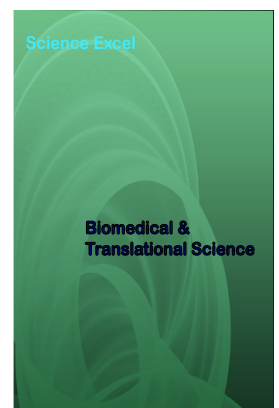

Correspondence

Ross Hansen

633 Idlewild Circle, Apartment, A2, Birmingham, AL 35205, USA.

Tel:+1 864-905-5263

E-mail: rosshansen@uabmc.edu

- Received Date: 21 Feb 2020;

- Accepted Date: 29 Feb 2020;

- Publication Date: 06 Mar 2020.

\section{Keywords}

Carcinoid syndrome, valvular disease, carcinoid heart disease, intrapulmonary shunt

\section{Abbreviations}

NET: neuroendocrine tumor; TTE: transthoracic echocardiogram; TEE: transesophageal echocardiogram; RHC: right heart catheterization; PFO: patent foramen ovale; RV: right ventricle; AVM: arteriovenous malformation

Copyright

(C) 2021 Science Excel. This is an openaccess article distributed under the terms of the Creative Commons Attribution 4.0 International license.

\title{
Right and Left-sided Valve Disease: A Case of Subclinical Intrapulmonary Shunt in an Adult with Malignant Carcinoid
}

\author{
Ross M Hansen ${ }^{1 *}$ and Gregory D Chapman ${ }^{2}$ \\ 'Tinsley Harrison Internal Medicine Residency, University of Alabama at Birmingham, USA \\ ${ }^{2}$ Department of Cardiovascular Disease, University of Alabama at Birmingham, USA
}

\begin{abstract}
Carcinoid tumors are highly differentiated neuroendocrine tumors (NET) that most commonly originate from the gastrointestinal tract. Liver metastases bypass first-pass metabolism and liberate vasoactive hormones into systemic circulation, causing flushing and diarrhea. Prolonged levels of circulating serotonin may adversely affect the heart by creating fibrotic endocardial deposits on native valves. The remaining serotonin is metabolized in the pulmonary circuit that leads to pathognomonic valvular disease isolated to the right side of the heart. We present a case of an adult male with known carcinoid syndrome who presented with involvement of right, as well as left-sided valves. He was found to have an intrapulmonary shunt on transesophageal echocardiogram (TEE) with bubble study. Intrapulmonary shunt should be considered, in conjunction with right-to-left shunt, lung involvement, and high levels of serotonin, for carcinoid patients with right and left-sided valve disease.
\end{abstract}

\section{History of Presentation}

A 55 year-old white male with known metastatic carcinoid presented to the university ED with the complaint of several weeks of worsening shortness of breath (SOB). His carcinoid symptoms (e.g. flushing, SOB) had been progressing for several months despite scheduled Sandostatin infusions with medical oncology. Beyond these symptoms, he endorsed several months of heart failure symptoms, including paroxysmal nocturnal dyspnea, abdominal swelling and decreased appetite.

On presentation to our ER, he was afebrile, BP $107 / 77$ tachycardic to HR 152, with $16 \mathrm{BPM}$ and saturating $96 \%$ on ambient air. On exam he was a middle-aged male in NAD, JVD was present, tachycardic but regular rhythm with grade III/ VI holosystolic murmur loudest at the left lower sternal border, clear lungs, soft and nontender abdomen, and no lower extremity edema.

\section{Past medical history}

His past medical history included the diagnosis of neuroendocrine tumor (NET) in the ascending colon 20 years earlier. He underwent a successful hemi-colectomy with surgical oncology. At the time of his surgery, he was not found to have distant metastases. But in the following years, he recurred with liver metastases, worsening flushing and diarrhea. He was placed on longacting Sandostatin infusions every 3 weeks with routine imaging surveillance with medical oncology. Furthermore, he had a 15 pack-year history of smoking and paroxysmal atrial fibrillation..

\section{Clinical Investigations}

An ECG confirmed 2:1 atrial flutter. He was imaged with transthoracic echocardiogram (TTE) demonstrating severe tricuspid and pulmonic regurgitation with severely reduced RV systolic function (Figure 1). To our surprise, the echocardiogram also demonstrated moderate mitral valve stenosis with rheumatic features (Figure 2). This prompted a discussion about concomitant diagnoses of rheumatoid and carcinoid heart disease. Later imaging with transesophageal echocardiogram (TEE) corroborated findings of rheumatic valvular disease. Namely, the mitral valve was thickened, retracted, and severely stenotic with moderate regurgitation. But bubble study showed the late passage of bubbles into the left atrium, indicative of intrapulmonary shunting (Figure 3).

His PET CT-Dotatate scan did not show myocardial involvement (Figure 4). $\mathrm{He}$ had relatively normal left-sided filling pressures on right heart catheterization (RHC) and preserved cardiac index of 2.3 with Fick physiology (Table 1). Coronary angiography showed a $70 \%$ stable obstruction of OM1 but otherwise non-obstructive coronary artery disease.

\section{Differential Diagnosis}

The differential for left-sided valvular disease included PFO, bronchial involvement, extensive burden of carcinoid from distant metastases to the liver, intrapulmonary shunt, or concomitant rheumatic mitral valve disease. 


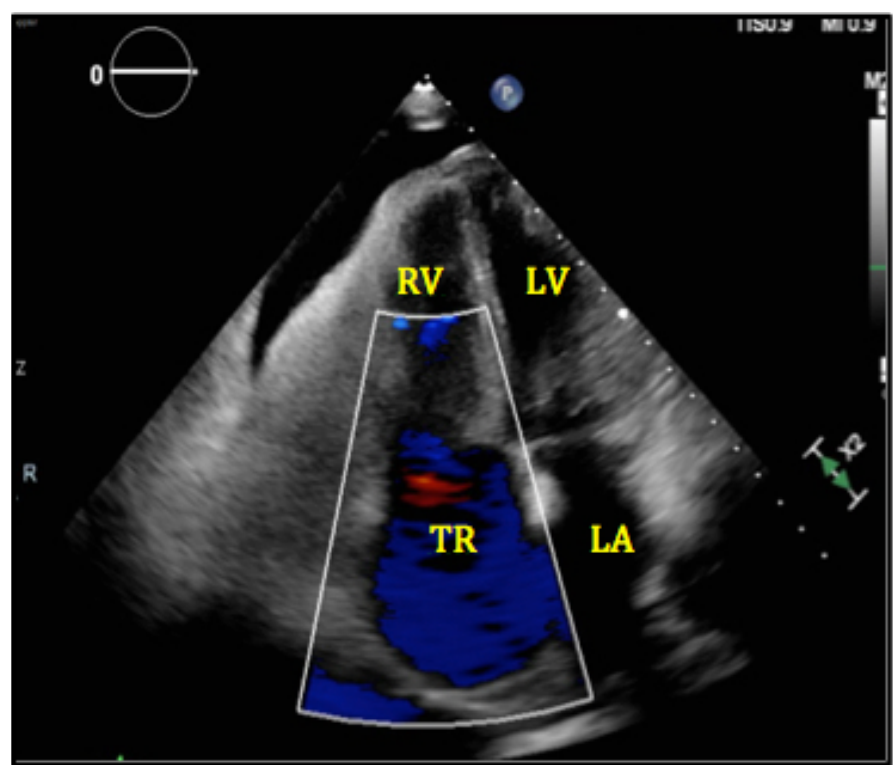

Figure 1: Severe Tricuspid Regurgitation (STR) noted on apical four chamber view with transthoracic echocardiogram (TTE).

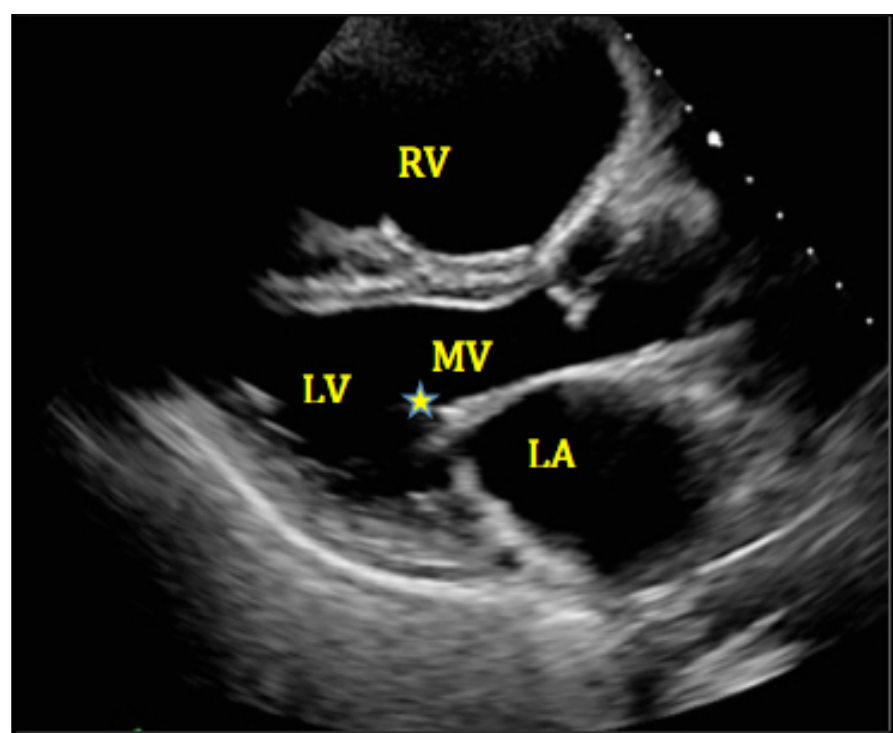

Figure 2: Parasternal long view with evidence of a thickened mitral valve (MV).

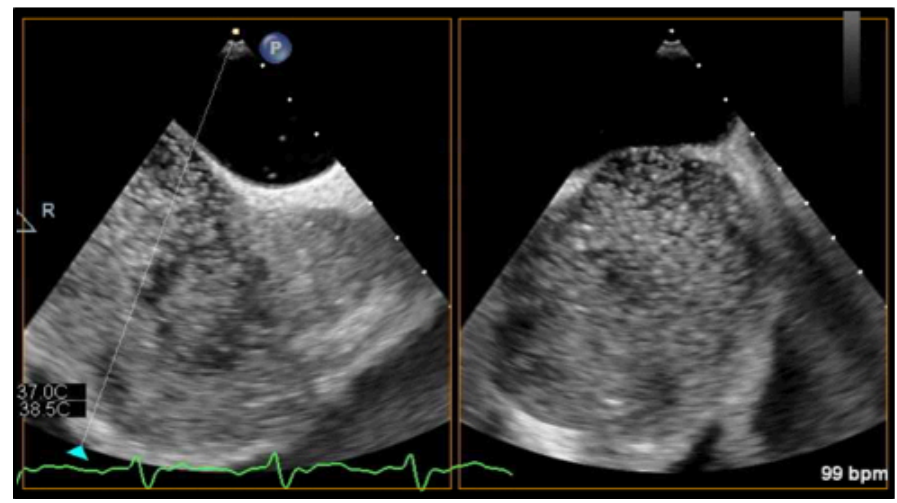

Figure 3: TEE showing the late passage of bubbles on bubble study suggestive of intrapulmonary shunt. Interatrial septum without evidence of atrial septal defect (ASD).
Figure 4: PET CT Dotate body scan demonstrated diffuse increase in radiotracer activity noted in liver (SUB max 26.5) consistent with metastatic carcinoid and expected physiologic update in the spleen and urinary bladder.

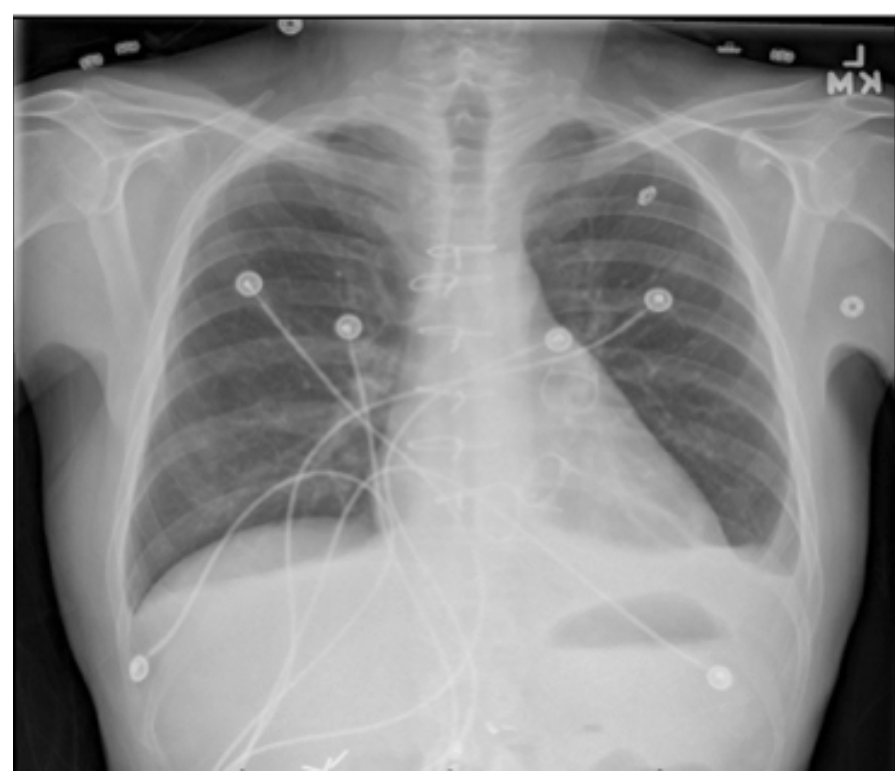

Figure 5: Posterior-anterior (PA) film of the chest with postoperative evidence of sternotomy and triple valve replacement of the tricuspid, pulmonic, and mitral valves. 
Table 1: Pre-operative intracardiac pressures measured with right heart catheterization (RHC).

\begin{tabular}{|l|l|l|l|l|}
\hline & \multicolumn{4}{|c|}{ Pressure, mmHg } \\
\hline & Systolic & Diastolic & End-Diastolic & Mean \\
\hline Right Atrium & & & & $5(2-6)$ \\
\hline Right Ventricle & $32(15-25)$ & & $2(0-8)$ & \\
\hline Pulmonary Artery & $27(15-25)$ & $11(8-15)$ & & $19(10-20)$ \\
\hline $\begin{array}{l}\text { Pulmonary Capillary } \\
\text { Wedge }\end{array}$ & & & & $12(6-12)$ \\
\hline
\end{tabular}

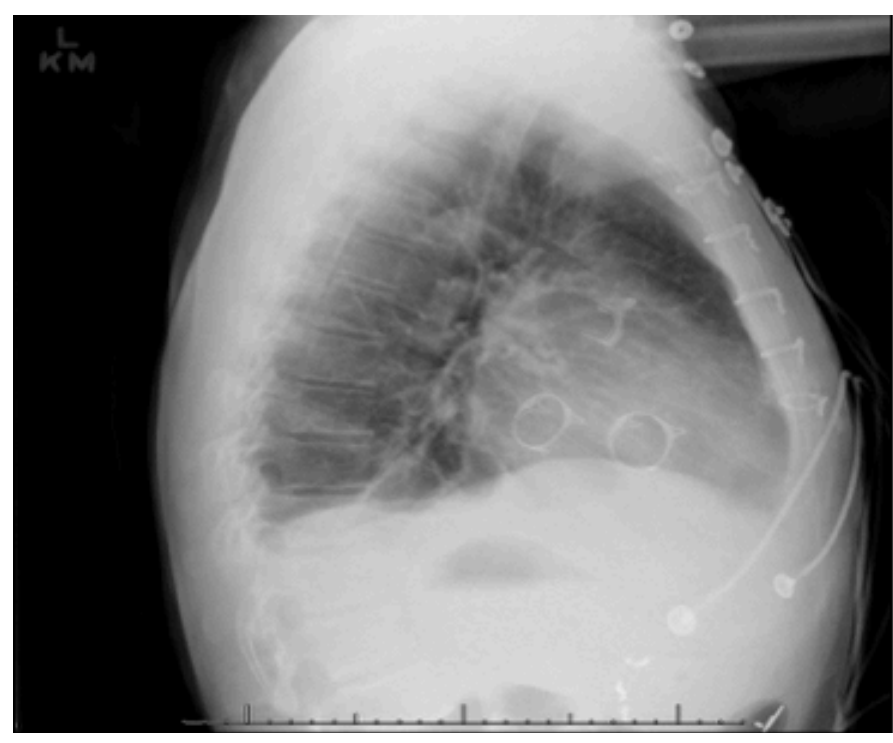

Figure 6: Lateral plane film of the chest with post-operative evidence of sternotomy and triple valve replacement of the tricuspid, pulmonic, and mitral valves

\section{Management}

Heart rate control with beta-blockade was attempted. It was felt that his atrial flutter was from significant atrial dilation and RV failure, so cardioversion and ablation were deferred. He was transitioned to short-acting octreotide (150 mcg TID) while inpatient and volume optimized with diuretics. CV surgery with triple valve replacement was advised. On hospital day 14, he underwent triple valve replacement with all tissue valves. He was bolused with octeotide (500 $\mathrm{mcg}$ ) two hours prior to surgery with an intra-operative infusion. Post-operatively, he was managed in the CICU for nine days where he was quickly weaned from inotropic support and intra-aortic balloon pump (IABP). Myocardial biopsies and pericardial fluid cytology were negative for malignancy. He subsequently returned home on hospital day 27.

\section{Discussion}

We present a case of a middle-aged male both right and left-sided cardiac involvement due to an intrapulmonary shunt. Through unclear mechanisms, carcinoid syndrome creates fibrotic endocardial deposits, predominantly along the tricuspid and pulmonic valves [1]. Prolonged exposure to serotonin may lead to retraction of native valves, non-coaptation, and ultimately stenosis and regurgitation [1]. Tricuspid regurgitation is perhaps the most common presentation with estimates greater than $90 \%$ for patients with cardiac involvement $[1,3]$. Left-sided cardiac involvement is less common, due to metabolism of serotonin in the pulmonary circuit, with estimates less than $10 \%$ of affected populations [2]. Pathologic explanations for left-sided involvement include carcinoid involvement of the lung, a right-to-left intracardiac shunt, or high systemic burden of serotonin, histamine, tachykinins, or prostaglandins. This may be seen with higher urinary levels of 5-HIAA [1-3]. Additionally, there are several reports documenting advanced valvular disease in patients with active use of SSRIs in carcinoid syndrome [4].

In the case of our patient, the late passage of bubbles with agitated saline on TEE indicated intrapulmonary shunting over an intracardiac shunt. It has been proposed in some cases that severe right-sided disease may cause right atrial pressures to exceed left atrial pressures, creating a right to left intracardiac shunt by reopening a previously closed PFO [5]. For our patient, it seems more likely that he carried a congenital arteriovenous malformation (AVM). He was also not hypoxic on vitals. Perhaps most interesting, his TTE on admission initially confounded his final diagnosis of intrapulmonary shunt, as there were features of rheumatic mitral valve disease. This prompted discussions about concomitant diagnoses of rheumatic and carcinoid heart disease. But a TEE later in his admission showed delayed passage of bubbles with agitated saline, favoring a unified diagnosis of triple valve involvement from carcinoid heart disease.

Our case is unique for several reasons. First, our patient presented with a sub-clinical intrapulmonary shunt later found on TEE by late passage of bubbles with agitated saline. This highlights the importance of maintaining a broad differential for left-sided valvular disease in patients with known carcinoid. Cardiac involvement in carcinoid syndrome portends a worse prognosis and overall mortality with an estimated three-year survival is $31 \%$ with cardiac involvement compared to $68 \%$ [2]. When identified, surgery has shown to improve morbidity with post-operative reductions in NYHA heart failure class [6]. The second unique feature of this case was early imaging findings that confounded the final diagnosis of intrapulmonary shunt. His initial TTE showed evidence of rheumatic heart disease and mitral stenosis, raising the question of concomitant diagnoses of rheumatic and carcinoid heart disease. His later TEE confirmed a unifying diagnosis of right and left-sided carcinoid heart disease by means of intrapulmonary shunt.

\section{Follow-up}

His post-operative course was uncomplicated. He returned home with the assistance of home health, completed a 2 months course of coumadin, and continued previously scheduled Sandostatin infusions every three weeks with oncology.

\section{Conclusion}

Left-sided valvular disease in uncommon in carcinoid syndrome. Echocardiographic findings of the mitral valve may share similarities with those seen in rheumatic heart disease. But as evidenced by delayed passage of bubbles on TEE, subclinical intrapulmonary shunt should be considered in the differential of those patients who have left-sided valve involvement. 


\section{References}

1. Yuan SM. Valvular Disorders in Carcinoid Heart Disease. Braz J Cardiovasc Surg. 2016; 31(5): 400-405.

2. Fox DJ, Khattar RS. Carcinoid heart disease: presentation, diagnosis, and management. Heart. 2004;90(10):1224-1228.

3. Pellikka PA, Tajik AJ, Khandheria BK, et al. Carcinoid heart disease. Clinical and echocardiographic spectrum in 74 patients.
Circulation. 1993;87(4):1188-1196.

4. Michalowska IM, Kolasinska-Cwikla A, Cwikla J, Zakrzewski D, Peczkowska M. Right and left-sided carcinoid heart disease. Cardiol J. 2018;25(3):417.

5. Bhattacharyya S, Davar J, Dreyfus G, Caplin ME. Carcinoid heart disease. Circulation. 2007;116(24): 2860-2865.

6. Mujtaba SS, Clark S. Quadruple Valve Replacement for Carcinoid Heart Disease. Braz J Cardiovasc Surg. 2018;33(4):398-403. 